\title{
Scalpel debridement has minimal effects on painful plantar calluses in older people: a randomised trial
}

\author{
Karl B Landorf ${ }^{1,2^{*}}$, Adam Morrow', Martin J Spink², Chelsey L Nash, Anna Novak', Adam R Bird ${ }^{1,2}$, Julia Potter ${ }^{3}$, \\ Hylton B Menz ${ }^{2}$
}

From 3rd Congress of the International Foot and Ankle Biomechanics Community

Sydney, Australia. 11-13 April 2012

\section{Background}

Plantar calluses are often associated with increased plantar pressure and foot pain, which can have a detrimental impact on the mobility and independence of an older person. Scalpel debridement is a key management strategy for painful calluses; however the effectiveness of this treatment in older people has not been rigorously investigated. The aim of this randomised trial was to evaluate the effectiveness of scalpel debridement in reducing plantar pressure and pain associated with forefoot plantar calluses.

\section{Materials and methods}

Eighty participants aged 65 years and older with painful forefoot plantar calluses were recruited. Participants were randomly allocated to one of two groups: (i) normal scalpel debridement or (ii) sham (control) scalpel debridement. Participants were followed for six weeks. Both participants and assessors were blinded to the intervention. The primary outcomes measured were the difference between groups in pain (100 mm VAS) and barefoot plantar pressure (MatScan ${ }^{\circledR}$ System).

\section{Results}

Both groups experienced large decreases in pain following intervention (up to a $41.9 \mathrm{~mm}$ decrease in pain on a VAS). A systematic, but small beneficial effect on pain was noted in favour of the normal scalpel debridement group immediately post-debridement to 4 weeks post debridement (from 6.0 to $7.2 \mathrm{~mm}$ ANCOVA adjusted mean difference between groups). These values, however, are unlikely to be clinically important to a patient and there were no statistically significant differences $(P<0.05)$ at any of the primary endpoints (immediately, and at 1, 3 and 6 weeks post-debridement). There were no statistically significant differences in plantar pressure between the two groups at any time-points.

\section{Conclusions}

The findings of this trial indicate that scalpel debridement of painful plantar calluses has minimal effect. While we found a systematic effect favouring scalpel debridement, the benefits were small and not statistically significant. There was no change in plantar pressure following scalpel debridement.

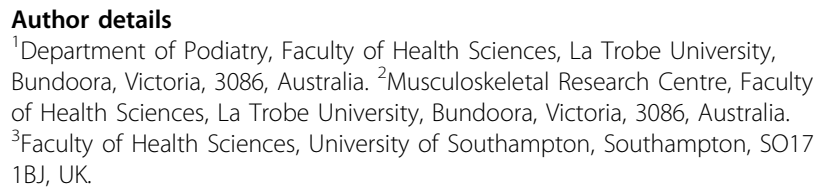

Published: 10 April 2012

doi:10.1186/1757-1146-5-S1-O27

Cite this article as: Landorf et al: Scalpel debridement has minimal effects on painful plantar calluses in older people: a randomised trial. Journal of Foot and Ankle Research 2012 5(Suppl 1):O27.

\footnotetext{
* Correspondence: k.landorf@latrobe.edu.au

'Department of Podiatry, Faculty of Health Sciences, La Trobe University,

Bundoora, Victoria, 3086, Australia

Full list of author information is available at the end of the article
}

(c) 2012 Landorf et al; licensee BioMed Central Ltd. This is an Open Access article distributed under the terms of the Creative Commons 